\title{
Manufactura inteligente y logística como un medio del desarrollo de pequeñas y medianas empresas
} Smart manufacturing and logistics..............................................
as a means of developing small and
medium-sized enterprises

\section{Laura Patricia Carranza Murillo*}

(C) Recepción: 02/09/2019

(C) Aprobación: 06/11/2019

(C) Publicación: 19/12/2019

\section{Para citar este artículo:}

D. Carranza, Murillo, L. P. (2019). Manufactura inteligente y logística como un medio del desarrollo de pequeñas y medianas empresas. Indagare, (7), 62-67. https://doi.org/10.35707/indagare/708

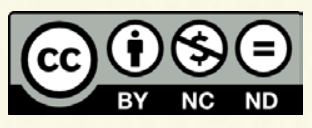

* Grupo de investigación GINNOVA, Universidad de Ibagué, ORCID: 0000-0002-7025-1417, correo electrónico: laura. carranza@unibague.edu.co 


\title{
Resumen
}

En el marco de la XI Escuela Internacional de Verano, la revista Indagare habló con el doctor Helmut Zsifkovits acerca su experiencia de trabajo en conjunto con la Universidad de Ibagué, a través de la asignatura Smart Logistics and Supply Chain. El doctor Zsifkovits nos comentó sobre de la importancia de estas temáticas en el desarrollo de pequeñas y medianas empresas, su participación en proyectos de investigación en el ámbito internacional y programas relacionados con la movilidad estudiantil y formación de lazos de colaboración con universidades en Colombia y Latinoamérica.

\begin{abstract}
Within the framework of the XI International Summer School, Indagare magazine spoke with Dr. Helmut Zsifkovits about his experience working together with Universidad de Ibagué, through the subject Smart Logistics and Supply Chain. Dr. Zsifkovits told us about the importance of these issues about the development of small and mediumsized enterprises, their participation in international research projects and programs related to student mobility and partnerships formation with universities in Colombia and Latin America.
\end{abstract}

Palabras claves: Gestión de cadenas de suministro, manufactura inteligente, lazos de colaboración, movilidad estudiantil internacional.

Key words: Supply chain management, smart manufacturing, partnerships, international student mobility.

\section{Introducción}

La industria global ha evolucionado bajo un nuevo paradigma. Ya no es suficiente producir más rápido, a menor costo y con una mayor calidad respecto a los competidores, con el fin de mantener una ventaja comparativa. Es así como la adopción de tecnologías de información y comunicación a lo largo del ciclo de vida de los productos y servicios, conocida como Manufactura Inteligente (Smart Manufacturing), es considerada un medio para desarrollar una ventaja competitiva en las Pequeñas y Medianas Empresas (PYMES).

La revista Indagare entrevistó al profesor Helmut Zsifkovits, quien es miembro del consejo de administración de la Bundesvereinigung Logistik Österreich (BVL), una de las redes industriales más grandes de Austria, cuya actividad está dirigida al desarrollo 
y aplicación práctica de la logística mediante la promoción y el mantenimiento de un intercambio interdisciplinario de experiencias entre la práctica y la ciencia. Así mismo, se ha desempeñado como docente en varias universidades y colegios.

La preocupación personal del doctor Zsifkovits por la cátedra y su interés en temáticas relacionadas a la logística, la gestión de procesos y la gestión de la complejidad, lo han llevado a establecer relaciones de cooperación a través de las fronteras y participar en programas de movilización estudiantil y docente, publicando a su vez numerosos artículos sobre conceptos de logística y otros temas.

\section{Usted se encuentra dictando la asignatura Smart Logistics and Supply Chain ¿En qué consiste y a quienes está dirigida?}

Hemos trabajado con la Universidad de Ibagué por doce años en diferentes campos de la logística y en los últimos años se han realizado importantes cambios en esta temática, especialmente en el desarrollo y aplicación de nuevas tecnologías. Naturalmente, nos hemos enfocado en el estudio de estas nuevas tendencias en la logística. Actualmente la Montanuniversität Leoben, institución a la cual estoy adscrito, trabaja en un proyecto internacional que involucra a seis países: Italia, Eslovaquia, Austria, Tailandia, India y Estados Unidos. De acuerdo a estos temas, buscamos formar y extender esta red internacional, hablamos de cómo trabajar a futuro y cooperar haciendo investigación, y desarrollo de actividades de enseñanza juntos en este importante campo.

Tenemos entendido que usted se encuentra trabajando como coinvestigador en el proyecto: "Estudio de las relaciones colaborativas en una cadena de suministros: Un caso de aplicación a la Administración de Proyectos" adscrito a la Universidad de Ibagué ¿El proyecto internacional que nos menciona, se relaciona de alguna manera con el proyecto en el que usted participa en nuestra institución?

Correcto, precisamente tiene el mismo enfoque. El proyecto en el cual trabajamos en la Montanuniversität Leoben, titulado SME 4.0, investiga las oportunidades de aplicación de nuevas tecnologías para la integración logística, especialmente para las PYMES. Creo que la situación en Colombia se asemeja mucho para los casos de pequeñas empresas con mayores dificultades para acceder al conocimiento y a la tecnología en este campo de la logística.

\section{¿En qué tipo de industrias se podría aplicar este tipo de temática?}

Es posible aplicarlo en cualquier tipo de industria, principalmente en la industria manufacturera. Especialmente aquellas que generan algún tipo de productos y en menor medida, las empresas de servicios. Podría ser en industria automotriz, farmacéutica o agrícola. 
¿Existe alguna diferencia para usted en la aplicación de esta temática para productos de distribución nacional con los de exportación?

No realmente, nos referimos a la gestión de cadenas de suministro. Estas, son internacionales para la mayoría de los productos. Muchas cadenas de suministro atraviesan fronteras desde Sudamérica a Europa y otros lugares, haciendo de esta una temática internacional.

¿Qué rama de la industria ha llamado su atención en sus visitas a Colombia?

He tenido la oportunidad de conocer algunas compañías, y de acuerdo a mi concepto personal, el sector de la agricultura es muy fuerte. Aun así, las compañías relacionadas a este sector, las cuales proveen la maquinaria, también lo son, pues son capaces de llevar a cabo sus actividades de una forma muy organizada.

\section{¿Cuál es la importancia de establecer lazos de colaboración con universidades en} Colombia y Latinoamérica?

Según mi punto de vista, en este proyecto hemos conocido muchos países y culturas. Pasamos algunos meses en Estados Unidos y Tailandia, y creo que es muy importante entender las diferencias entre culturas e idiomas. En mi opinión, existe un gran potencial de colaboración entre Europa y Sudamérica. Por ejemplo, si se establecen negociaciones o lazos con China, encontramos que estos son muy pequeños en comparación con Europa, pero este país es una potencia económica. Por otro lado, Sudamérica tiene algunos lazos históricos y la cultura incluye varios puntos en común. Además, los lazos de colaboración son muy importantes para establecer programas de movilidad estudiantil y docente, ya que es más complicado para los estudiantes y profesores colombianos llegar a Europa. Es por eso que tratamos de buscar oportunidades financiadas a través de la Unión Europea, y así permitir que tanto estudiantes como profesores puedan desplazarse a Europa. Esto no debe ser una ruta de una sola vía.

Tuvimos la oportunidad de trabajar con European Region Action Scheme for the Mobility of University Students (ERASMUS), que es un programa de movilidad estudiantil financiado por la Unión Europea, que además incluye algunos países fuera de la Unión Europea. Se ha aplicado este proceso dos veces y desde hace dos años se ha obtenido financiación para intercambios.

\section{¿Qué perfil se requiere para acceder al programa ERASMUS?}

Existen dos niveles, el primero corresponde a los docentes, quienes usualmente viajan durante una o dos semanas al país y el segundo, a estudiantes que viajan durante un 
semestre. Hemos tenido estudiantes colombianos en Austria y estudiantes austriacos en Ibagué. Es un programa que se está iniciando, pero tratamos de mejorar cada vez más. Este procura seleccionar tanto a profesionales como a estudiantes de semestres más avanzados, siempre y cuando posean los conocimientos básicos necesarios para el intercambio. El número de intercambios es limitado y el acuerdo con los estudiantes que van a Austria consiste en pagar una tarifa de viaje, pero el ingreso a la universidad es gratuito. Los gastos de vivienda y alimentación dependen de los fondos disponibles en el programa ERASMUS, usualmente estos gastos están cubiertos para las pasantías de docentes, pero se espera que a medida que el Programa avance se puedan cubrir los gastos de algunos estudiantes.

\section{¿Cuál ha sido su experiencia al estar vinculado con la Universidad de Ibagué?}

El vínculo establecido con la Universidad de Ibagué inició hace 15 años, cuando uno de sus profesores (un vicerrector) visitó Austria para una reunión de internacionalización de redes, así que nos pusimos en contacto para conversar acerca de posibles intercambios. Nos tomó alrededor de tres años antes de que todo tomara forma e iniciara, y se desarrolló una relación de colaboración que no ha parado. Estoy muy feliz de comentar, además, que tuve la oportunidad de trabajar con uno de sus docentes, Carlos Meisel, quien estuvo en Austria por algunos años.

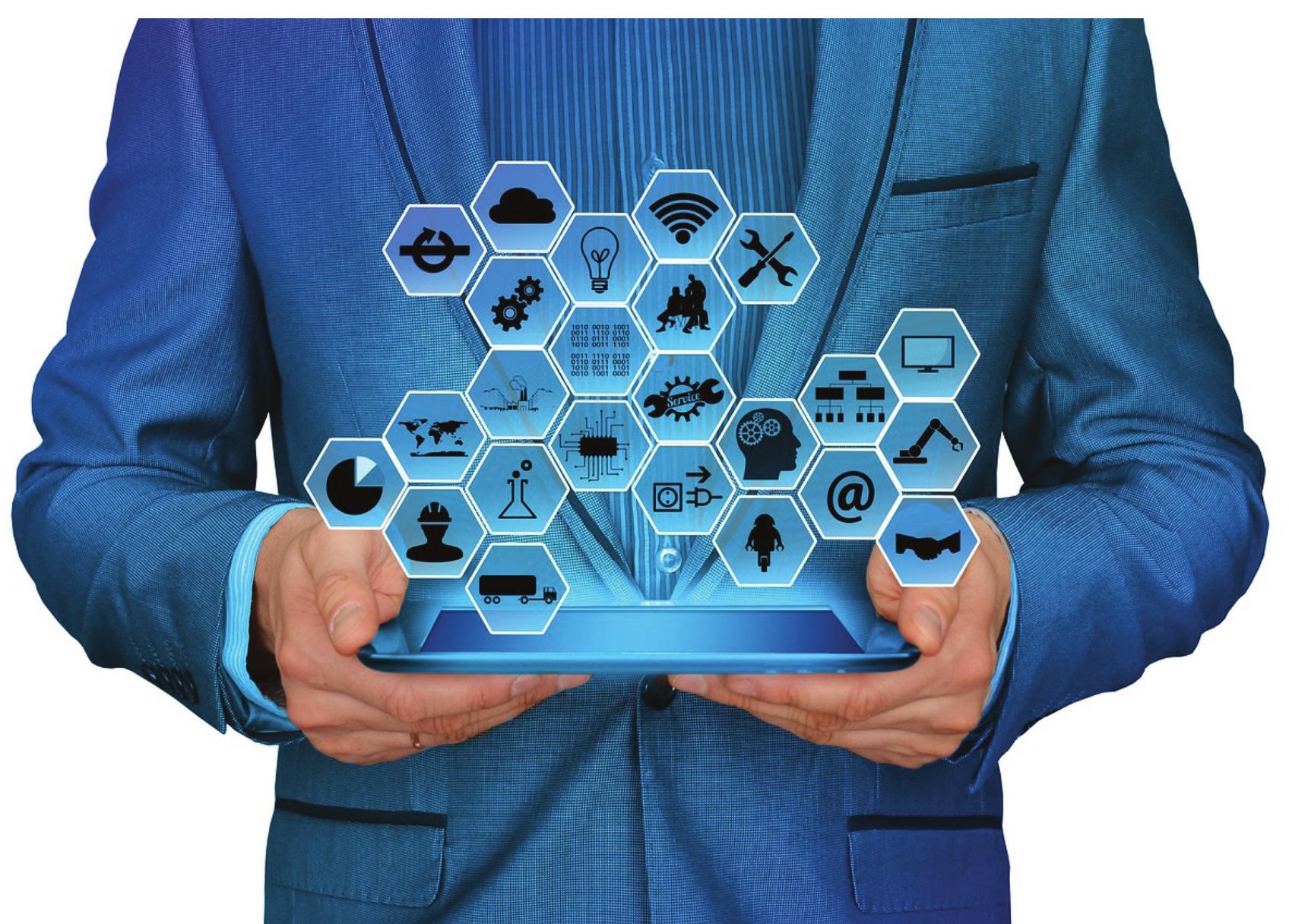


He sentido que realmente existe una actitud más abierta y de amistad con las personas con las que he hablado, comparado con otros países que mencioné anteriormente. He estado algunos meses en los Estados Unidos, pero aquí es mucho más fácil hablar con las personas para mantenerse en contacto y establecer acciones a futuro. Esta es mi opinión y mi convicción.

El desarrollo de las tecnologías de comunicación e información, sumado a un entorno globalizado, ha permitido que las cadenas de suministro atraviesen fronteras y se formen alianzas de carácter internacional a través de los diferentes actores que la conforman. Aun así, esta expansión en el concepto de cadenas de suministro ha generado la necesidad de estudiar las nuevas tendencias en la logística, las cuales integran la tecnología como una herramienta clave en la gestión de dichas cadenas. Cabe resaltar que el ejercicio de investigación que surge de esta necesidad requiere a su vez de la formación de relaciones de colaboración entre investigadores, docentes y estudiantes de diferentes entornos y culturas, dado que esta diversidad profesional y cultural permite una mayor comprensión acerca de la gestión de las cadenas de suministro en diferentes sectores de la industria y en entornos socio-culturales.

Agradecemos al doctor Zsifkovits por participar de esta entrevista y compartir sus pertinentes reflexiones acerca de las actuales brechas investigativas en el campo de la logística y la gestión de cadenas de suministro. Así mismo, invitamos a la comunidad educativa a explorar las oportunidades que ofrecen este tipo de vínculos académicos, puesto que pueden servir como puentes en procesos de movilidad estudiantil en los diferentes niveles académicos.

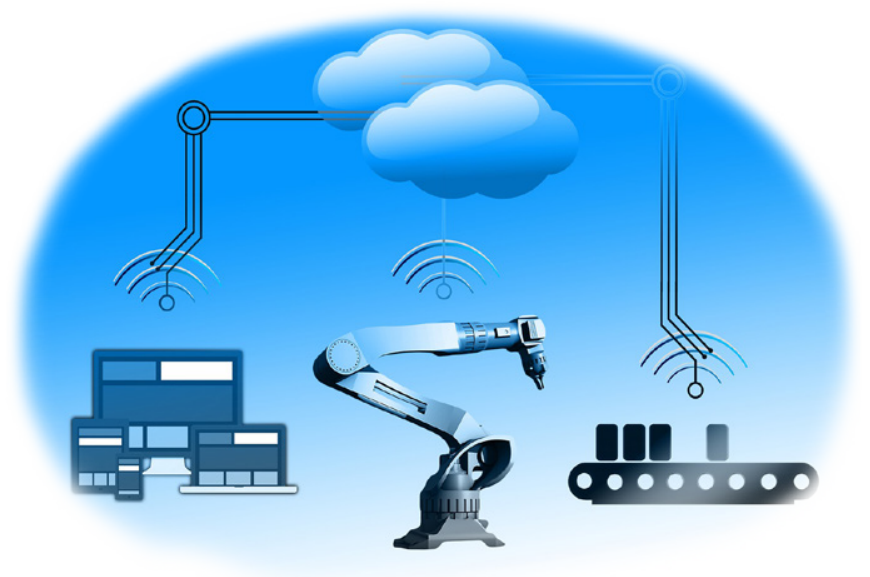

